\section{Built on trust}

\section{Written agreements between parties in research collaborations are not a sign of a lack of faith.}

A scuffle that has riled the Chinese scientific community could have been avoided if the parties involved had hammered out the details of their collaboration beforehand.

On 16 November, researchers at Peking University in Beijing claimed discovery of a biological-compass mechanism that could explain how some animals sense magnetism (S. Qin et al. Nature Mater. http://doi.org/89v; 2015). But some of the paper's thunder was stolen by a researcher at Tsinghua University, also in Beijing, who reported in September how the same mechanism could be used to manipulate neurons in worms (X. Long et al. Sci. Bull. http://doi.org/883; 2015).

When the September paper was published, the lead Peking University researcher cried foul, claiming that his Tsinghua colleague had agreed not to publish until the Nature Materials paper came out (see Nature http://doi.org/9gg; 2015). University administrators got involved, the Tsinghua researcher was fired, and his graduate student, whose career has been upended, circulated a plea for support to China's scientific community. The Peking researcher has called for his rival's paper to be retracted. Both parties have mustered e-mails and other correspondence to show that the facts are on their side.

A detailed, formalized agreement could have prevented this. When embarking on a collaboration, it can be hard to ask a scientific peer to sign a contract. Lawyers get involved, making it cumbersome and costly. Fencing off rights to patents, authorship, publication and decision-making authority can be tedious and can cause tension. A simple handshake is much more comfortable.

This is true for researchers around the world. But in China, where people are finely tuned to what might make them lose face, the bar is especially high. Asking someone to sign such an agreement feels equivalent to saying that you don't trust them.

A survey of Chinese researchers undertaken by Nature Publishing Group supports that observation. Scientists who had worked abroad
"The biggest hindrance to harmonious collaboration was tension over authorship." were asked about the differences in the working environment in China compared with that in other countries, including the ease of carrying out collaborations. Some noted that Chinese researchers usually do not ask for formal agreements. The reason might be cultural, but it could also be that most universities and research organizations in China do not have the personnel to support this function.

The survey results appear in a 26 November report, Turning Point: Chinese Science in Transition (see go.nature.com/ybsatt and go.nature. com/fdwacj; in Chinese). The biggest hindrance to harmonious collaboration, according to interviewees, was tension over authorship - a factor that plays a substantial part in the dispute over the biological-compass papers. In China, assessors of a researcher's achievements focus on papers in which the individual is first or corresponding author. The report suggests that research assessment should take a more balanced approach, and that policymakers can iron out some of these wrinkles.

It is clear that university administrators can help collaborations by providing personnel to deal with the legal aspects. It might be a burden in the short term, but in the long term it would encourage collaboration. Scientists with valuable knowledge who want to protect their rights to priority in publication, patents and other areas deserve as much.

\section{Drugs on demand}

\section{Controversy in Brazil over access to a purported cancer cure could set a harmful precedent.}

A furious debate that is raging in Brazil pits the nation's largest university against hundreds of cancer patients who want access to a compound that some have branded a miracle cure. But whether the compound holds any benefits at all remains to be seen: it has never been evaluated in human trials. The conflict is an extreme version of a debate that has gone on in the United States and elsewhere, as terminally ill people whose diseases have withstood modern medicine's proved arsenal have demanded access to untested treatments.

As we report on page 420, courts in Brazil have previously sympathized with those demands, ordering the University of São Paulo to provide a compound called phosphoethanolamine to hundreds of patients. People on both sides of this debate are armed with good intentions. The university argues that the drug is untested, and should not be used to give false hope - and unknown side effects - to vulnerable patients. On the other side, it is understandable that people with little hope may prefer the uncertainty of an untested drug to the certainty of a terminal illness.

But there are also concerning reports that some people with cancer are not taking their prescribed medications, for fear that scientifically proven medicine may interfere with the supposed miracle of phosphoethanolamine. The tenor of the debate has also been harmful at times, with some phosphoethanolamine advocates accusing the government or the pharmaceutical industry of actively suppressing further development of the drug.

The sad truth is that the drug is unlikely to be a miracle. In the United States, for example, only one in ten drugs that make it to phase I clinical trials are destined to gain approval from the US Food and Drug Administration (FDA). And phosphoethanolamine has not even made it that far: its promise is backed up by a few publications based on lab and animal tests.

Even so, terminally ill patients may be willing to try a treatment with only the slimmest odds of success. In the United States, several states have passed laws that, to varying degrees, grant such patients the right to try experimental drugs outside the purview of the FDA. The laws have triggered debates of their own, and have come under fire for offering false hope and for potentially leading patients away from other, more promising avenues.

The situation in Brazil is more extreme. A university laboratory is neither a pharmaceutical plant nor a pharmacy; it is not required to follow good manufacturing protocols. There is no oversight to certify what is going into the blue-and-white phosphoethanolamine capsules produced at the University of São Paulo. Neither the compound's side effects nor its efficacy are systematically monitored. To order a university to supply a drug is to show a disregard for the importance of all these safety measures.

The hope of phosphoethanolamine lies in further research. Federal funders in Brazil have said that they will support further preclinical studies of the drug. Researchers are pursuing options for moving the compound into clinical trials, should those animal studies succeed; patients who are interested in pursuing phosphoethanolamine
SNATURE.COM To comment online, click on Editorials at: go.nature.com/xhunqv treatment could enrol in the clinical tests. In the meantime, the courts should liberate patients from the legal tug-of-war and uphold the latest decision to halt distribution of phosphoethanolamine until its potential is better understood. 